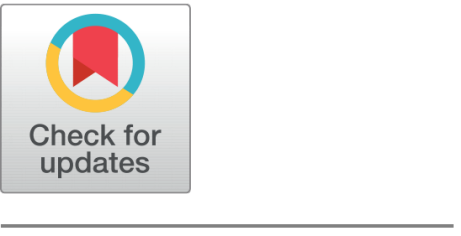

OPEn ACCESS

Received: 12.08.2021

Accepted: 22.10 .2021

Published: 21.12 .2021

Citation: Sathiya Priya S, Sathiaseelan JGR (2021) Enhanced Retina Blood Vessel Segmentation by Super Resolution Generative Adversarial Networks based U-Net. Indian Journal of Science and Technology 14(43): 3246-3253. https ://doi.org/10.17485/IJST/v14i43.1502

* Corresponding author.

priya.it09@gmail.com

Funding: None

Competing Interests: None

Copyright: (c) 2021 Sathiya Priya \& Sathiaseelan. This is an open access article distributed under the terms of the Creative Commons Attribution License, which permits unrestricted use, distribution, and reproduction in any medium, provided the original author and source are credited.

Published By Indian Society for Education and Environment (iSee)

ISSN

Print: 0974-6846

Electronic: 0974-5645

\section{Enhanced Retina Blood Vessel Segmentation by Super Resolution Generative Adversarial Networks based U-Net}

\author{
S Sathiya Priya ${ }^{1}{ }^{*}$, J G R Sathiaseelan ${ }^{1}$ \\ 1 Department of Computer Science, Bishop Heber College, Affiliated to Bharathidasan \\ University, Trichy, Tamil Nadu, India
}

\section{Abstract}

Objectives: To improve quality of images from video capture under normal illumination through SMART system and the best performance in the task of retina blood vessel segmentation with minimize segmentation loss and recover high resolution feature and makes it possible to evaluate high resolution image. Methods analysis: Existing research were showed for spontaneous segmentation of retina blood vessel from fundus images through supervised and unsupervised techniques. On the other hand, most of the research absence in segmentation robustness and cannot enhance loss functions so that results of the segmentation have made lots of fake. In our research, supervise the value of segmentation loss functions for a number of iterations and supports measure the accuracy of Super Resolution Generative Adversarial Network (SRGAN) method in training process using DRIVE dataset. Findings: We enhanced the AUC of $0.9943 \%$, Sensitivity of $0.8352 \%$ and specificity of $0.9849 \%$ using through SRGAN-UNet method. We additionally applied overlap tile technique for validation which made it conceivable to segment high resolution with overall precision $0.9736 \%$. Novelty: Our proposed method to produce new-fangled, imitation occurrences of data that can pass for real data processing method that make high resolution images from experimental lower solution images based U-Net.

Keywords: Super Resolution Generative Adversarial Networks; Retina Blood Vessel Segmentation; UNet; overlaptile Technique; Glaucoma Disease; Sensitive Mirror Analyzer and Retina Tracker (SMART) system

\section{Introduction}

Retinal images contain rich relevant constructions, for example, retinal vascular designs that can give significant clinical data to the diagnosis of sicknesses like Glaucoma and retina hypertension. Along these lines, the precision of retinal vessel segmentation can be utilized as a significant marker for the conclusion of related infections. Nonetheless, manual segmentation of retinal vessels is a tedious errand, so we are attempting to figure out how to consequently section retina vessels. Retina vessel segmentation ${ }^{(1)}$ assumes 
a critical part helped screening, analysis, and treatment of hypertension and Glaucoma disease. A considerable measure of work have been accounted for over the most recent twenty five years for mechanized recognizing vessels in retinal fundus images. To tackle these issues, we zeroed in on an understudy community point recognition utilizing infrared eye images caught by back to camera and proposed precise location strategies by U-Net.

Consistently in our day by day life, we routinely take a look at the mirror. It will be that simple if an automated tomography scan of the human eye through the mirror is done. The client to be in a legitimate situation before the mirror so that necessary images of the eyes will be effortlessly noticed for Glaucoma manifestations utilizing through ${ }^{(2)}$ Sensitive Mirror Analyzer and Retina Tracker (SMART) framework. Our SMART framework will be not difficult to adjust by individuals, in actuality. A person's appearance may likewise change because of shade of eye sclera territory. To conquer the constraints of conventional eye acknowledgment in this specific circumstance, one procedure is to perform sclera district recognition and characterization.

The major objective of this research work is to enhance the image quality from low resolution to high resolution by applying Retina blood vessel segmentation using SRGAN-Unet with overlap-tile strategy.The SRGAN with UNET is used in Training data set and to boost up the performance, Overlap Tile Strategy is used in testing data set.

The overlap-tile strategy is that it has to be run separately for each patch. Also, it has to be done in parallel due to the overlapping patches. On the other hand, if the network is only seeing little context, then the localization accuracy is compromised. In order to minimize this issue, larger patches require more max-pooling layer. A more recent approach proposed a classifier that takes into account the various features of multiple layers. Good localization can also be achieved at the same time by using context.

A network-based training strategy is presented that uses SRGAN to efficiently utilize the available annotated samples. It achieves its goal by taking advantage of the flexibility of the network and the robust expanding path. Among the algorithm options for FCN-based medical image segmentation, the U-net structure is proposed. It provided a uniform approach for adding a feature map, which performed well in terms of biomedical image segmentation. In the training phase, the vessel features of the convolution kernels are extracted. The proposed method is then created that is the discriminator of SRGAN. The discriminator was used for generating and distributing pixel distribution reports. The generator was used for generated and analyzed segmentation loss data.

In this research, we concentrate first level of processing is segment of retina vessel with higher resolution utilizing our proposed technique. The basic SMART Segmentation (SMARTSeg) framework Figure 1. When the person stands in front of the mirror, the mirror captures the images particularly eyes. Which is the input for pre-processing stage. The retina blood vessel images are augmented for Super Resolution images using SRGAN method based U-Net. The images collected from DRIVE dataset and compared with captured image from camera. We proposed SRGAN method to permit UNet ${ }^{(3)}$ to learn increases that best improve the classifier. The model comprises two networks that are trained in a SRGAN process where one network produces synthetic images and the other network segregates between the real and synthetic images more than once.

The majority of the systems have predefined modules that we can use to expand image data prior to train the SRGAN-UNet method. ${ }^{(4)}$ Rather than the original images, these enlarged of images are utilized for training. Along these lines, while training the SRGAN-UNet model will see the original images and the enhanced images. Utilizing this method, we can improve quality of the image.

In this study, we examined and proposed a different kind of augmentation where we combine U-Net that segment image so rather than standard augmentation with SRGAN learns enlargements that the best reduce segmentation loss. Moreover, we implement overlap-tile technique for local processing in testing dataset. This technique generally used for enhance the image when output image pixel smaller than input image pixel.

For all the above, we measure segmentation performance on the testing dataset as the metric to compare these augmentation approaches. 


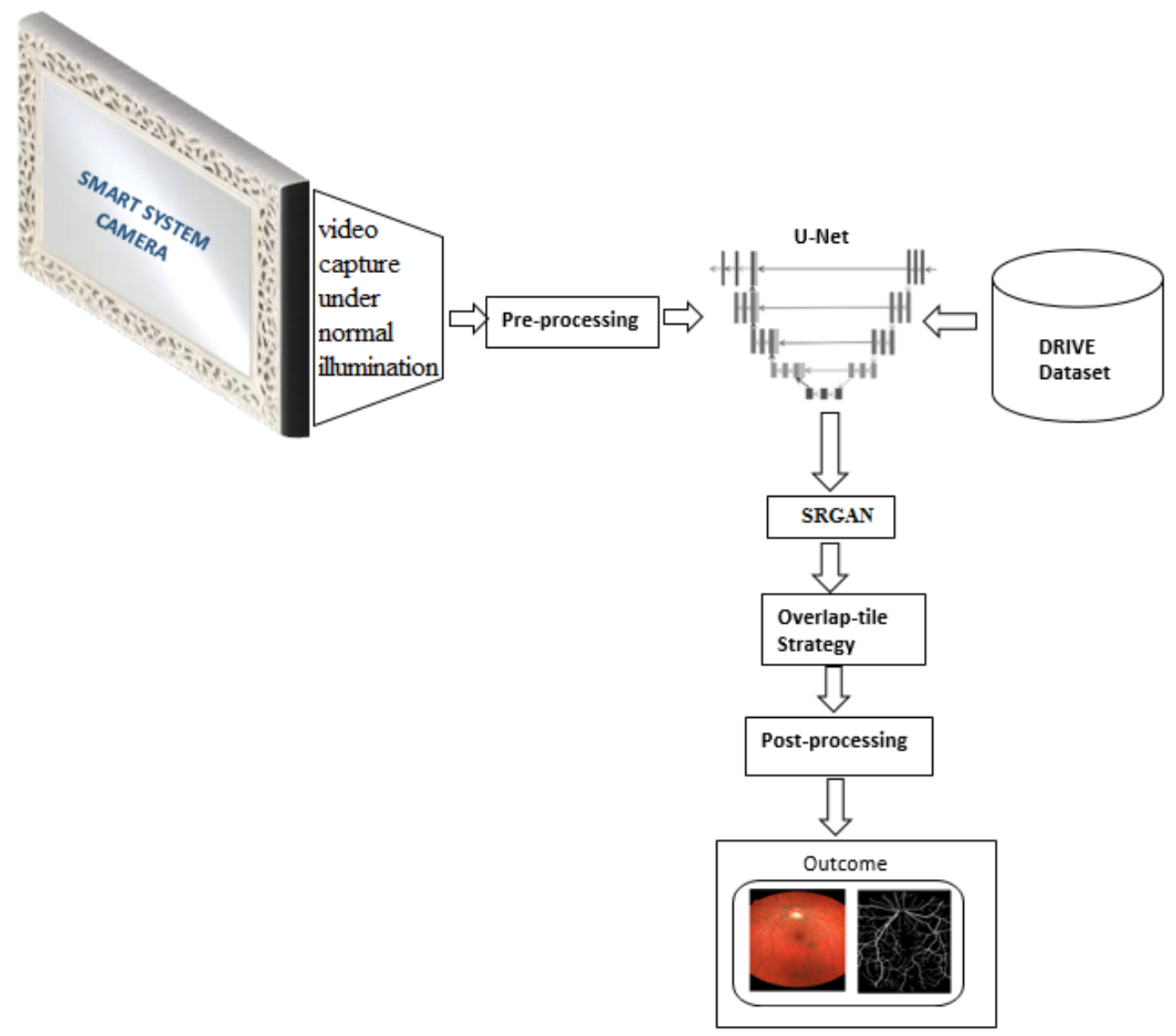

Fig 1. Basic SMARTSeg Framework

\section{Literature Review}

Recently, significant learning-based counts have continued to make and performed well in the field of retina vessel segmentation, which have constantly become the standard figuring. Existing studies were showed for spontaneous segmentation of retina blood vessel from fundus images through supervised and unsupervised techniques.

On the other hand, most of the research absence in segmentation robustness and cannot enhance loss functions so that results of the segmentation have made lots of fake ${ }^{(5)}$. Atli et al., ${ }^{(6)}$ planned robotized vessel segmentation and applied with up-sampling and down-sampling layers and looked at three sorts of data sets and their measurements.

Chen et al., ${ }^{(7)}$ investigated multi styles by relegating various loads to the gated change module with auto-encoder recreation misfortune. Chowdary et al., ${ }^{(8)}$ used the holomorphic channel joined with CLAHE technique for the enlightenment standardization and differentiation enlargement of the retinal images.

Guibas et al. ${ }^{(9)}$ proposed a fundus angiography image age technique, which can successfully improve the quality and variety of image age yet experiences loss of subtleties and cannot reduce images with comparing names.

Fan et al., ${ }^{(10)}$ produced tri-map naturally by used locale highlights of vessels, at that point applies a progressive image tangling model to remove the vessel pixels from the obscure districts.

Jin et al. ${ }^{(11)}$ obtainable Deformable U-net (DUNet) that uses the retinal vessel limited features with a U- net. $48 \times 48$ patches were also used for training dataset. Though, the patch-based segmentation takings more time in training and working out. In this research utilizing patches of a size $32^{\star} 32$ pixels. This fix size gave the best exactness of pixel arrangement by U-Net. Each resolution is acquired by arbitrarily choosing its middle inside the full image. 
To bear out the proposed approach, used visibly offered DRIVE ${ }^{(12)}$, CHASE-DB1 $1^{(13)}$, and STARE ${ }^{(14)}$ datasets and compared the proposed SRGAN with previous studies. We measured sensitivity, specificity, accuracy, F1-score, and AUC for comparative analyses. Comparative analyses showed that the proposed SRGAN-Unet derived higher performance than other studies.

\section{Methodology}

In this research, propose Super Resolution Generative Adversarial Networks based U-Net (SRGAN-U Net) method combined with Overlap-tile technique. It is mainly used for improve quality of images and the best performance in the task of image segmentation with reduce segmentation loss. It permits the network to recover full resolution feature -maps and makes it possible to analyze high resolution image.

\subsection{The Proposed Method: SRGAN-UNet}

The proposed method SRGAN is to generate new, synthetic instances of data that can pass for real data processing method that construct high resolution images from observed lower solution images based U-Net. We combined symmetric overlap-tile technique for local processing which made it probable to improve the accuracy. We refer to this technology as a post-processing method that only applied to the testing dataset. So finally, we get the outcome of accuracy too good compare to previous method. Our assessment arrangement Figure 2. First, we split the data into one or reformed training, approval, and test sets. At that point, we train SRGANs for each preparation set of this dataset.

\subsection{Overlap-tile Technique}

In this paper, cover tile system into the SRGAN technique to take care of the issue of under-segmentation at the edges of subimages. In this technique, increase size with quality from down-sampling pixel to up-sampling pixel.

The Overlap-tile technique ${ }^{(11)}$ was intended by O. Figure 2(A) this technique alluded this innovation to a piece of network so the extents of info and yield were not the equivalent for both the preparation and testing dataset. Segmentation consequence of basic local propagation technique Figure $2(B)$. The first image $B(1)$ indicates original image. The second image $B(2)$ indicates split technique addressed by gridlines. The last image $\mathrm{B}(3)$ indicates retina blood vessel segmentation consequence with under segmentation issues addressed by red circles. Figure 2(C) overlap-tile technique for continuous segmentation of random large images.

This optimization equation helps to increase the probability of the discriminator being able to differentiate between synthetic and real images and pushes the generator to create compelling real-like images to fool the discriminator more effectively.

The retina vessel images lower than down-sampled factor (r). So, we implement Gaussian filter for a blur smoothing effect in training dataset. This filter is owing to its frequency response.

Low quality pixel $R^{L}=\left(W * H * C^{n}\right)$

Super Resolution pixel $R^{H}=\left(r(W * H) * C^{n}\right)$

Here, 'W' illustrates Width, 'H' illustrates Height and $C^{n}$ illustrates number of color channels.

Standard Augmentation of real image $R_{S A}\left(x_{r}\right)=\sigma(C($ real $)) \rightarrow 1$

Standard Augmentation of synthetic image $R_{S A}\left(x_{s}\right)=\sigma(C$ (synthetic $\left.)\right) \rightarrow 0$

Above the formula represent to standard augmentation real and synthetic image is resultant image may be real or synthetic. It denotes ' 1 ' and ' 0 '.

Super Resolution GAN of Retinal image $R_{H a}\left(x_{r}, x_{s}\right)=\sigma(C($ real $)-\in[C($ synthetic $)]) \rightarrow 1$

Super Resolution GAN of Retinal image $R_{H a}\left(x_{s}, x_{r}\right)=\sigma(C$ (synthetic $)-\in[C($ real $\left.)]\right) \rightarrow 0$

The formula of Super Resolution GAN can be evaluate more realistic than synthetic data and less realistic than real data.

In training process, the model weights are iteratively in sync for that reason with the purpose of minimizing the segmentation loss.

$$
R_{S L}=-\frac{1}{N}\left[\sum_{i=1}^{N}\left[g t_{i} \log \left(p_{i}\right)+\left(1-g t_{i}\right) \log \left(1-p_{i}\right)\right]\right.
$$

Above the formula mainly calculated average segmentation loss, for $\mathrm{N}$ implies pixel points where $g t_{i}$ implies ground truth value taking a value 0 or 1 and $p_{i}$ is the probability for the $i^{\text {th }}$ pixel points. This equation helps to increase the probability of the discriminator being able to differentiate between synthetic and real images and pushes the generator to create absorbing real-like images to synthetic the discriminator more efficiently. 


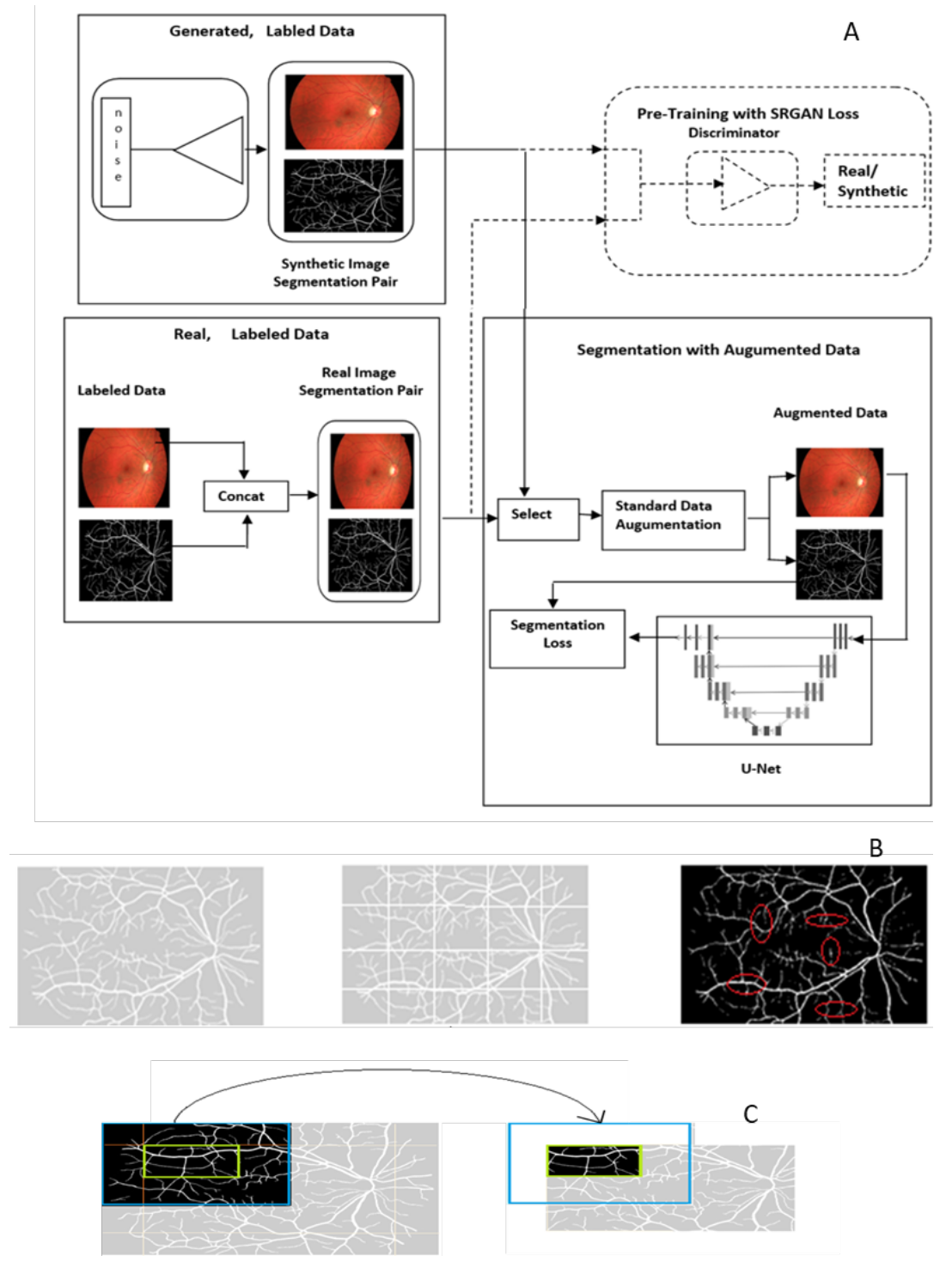

Fig 2. (A). Evaluation setup for SRGAN method architecture, (B). Segmentation consequence of basic local propagation technique. B(1) Original image; B(2) Split technique addressed by gridlines B(3) Retina Blood Vessel Segmentation consequence with under segmentation issues addressed by red circles. (C). Overlap-tile technique for Retina Blood Vessel Segmentation

\section{Implementation results}

\subsection{Dataset}

In this section, we select Datasets for Retina Blood Vessel image dataset through evaluation metrics. Table 1 Evaluation metrics of two different higher pixel rate retina blood vessel image datasets. F1 score and AUC also evaluate in DRIVE and CHASE_DB1 datasets. This result compared from previous methods and their metrics. Finally, DRIVE dataset slightly higher than others. The graphical representation of this result Figure 3(A).

Table 2 proposed SRGAN-Unet method and we show the result in this table and calculate F1 score, AUC, sensitivity, specificity, dice and accuracy. Figure 3(B), we represent graphical oriented performance quantitative result of our proposed method compare from previous methods. Our proposed method gives higher performance result compared with previous method. 
Table 1. Evaluation metrics of DRIVE and CHASE_DB1 dataset from Previous Methods

\begin{tabular}{llllll}
\hline Method & Year & Dataset & Model & F1 score & AUC \\
\hline \multirow{2}{*}{$(17)$} & 2020 & CHASE_DB1 & SA-UNet & 0.8153 & 0.9905 \\
& 2020 & DRIVE & SA-UNet & 0.8248 & 0.9860 \\
\hline \multirow{2}{*}{$(18)$} & 2019 & CHASE_DB1 & IterNet & 0.8073 & 0.9851 \\
& 2019 & DRIVE & IterNet & 0.8205 & 0.9816 \\
\hline \multirow{2}{*}{$(19)$} & 2018 & CHASE_DB1 & VGN & 0.8034 & 0.983 \\
& 2018 & DRIVE & VGN & 0.8263 & 0.9802 \\
\hline \multirow{2}{*}{$(11)$} & 2018 & CHASE_DB1 & DUNet & 0.7883 & 0.9804 \\
& 2018 & DRIVE & DUNet & 0.8237 & 0.9802 \\
\hline \multirow{2}{*}{$(20)$} & 2018 & DRIVE & LadderNet & 0.8202 & 0.9793 \\
& 2018 & CHASE_DB1 & LadderNet & 0.8031 & 0.9839 \\
\hline$(15)$ & 2019 & DRIVE & BCDU-Net (d=3) & 0.8224 & 0.9789 \\
\hline
\end{tabular}

A
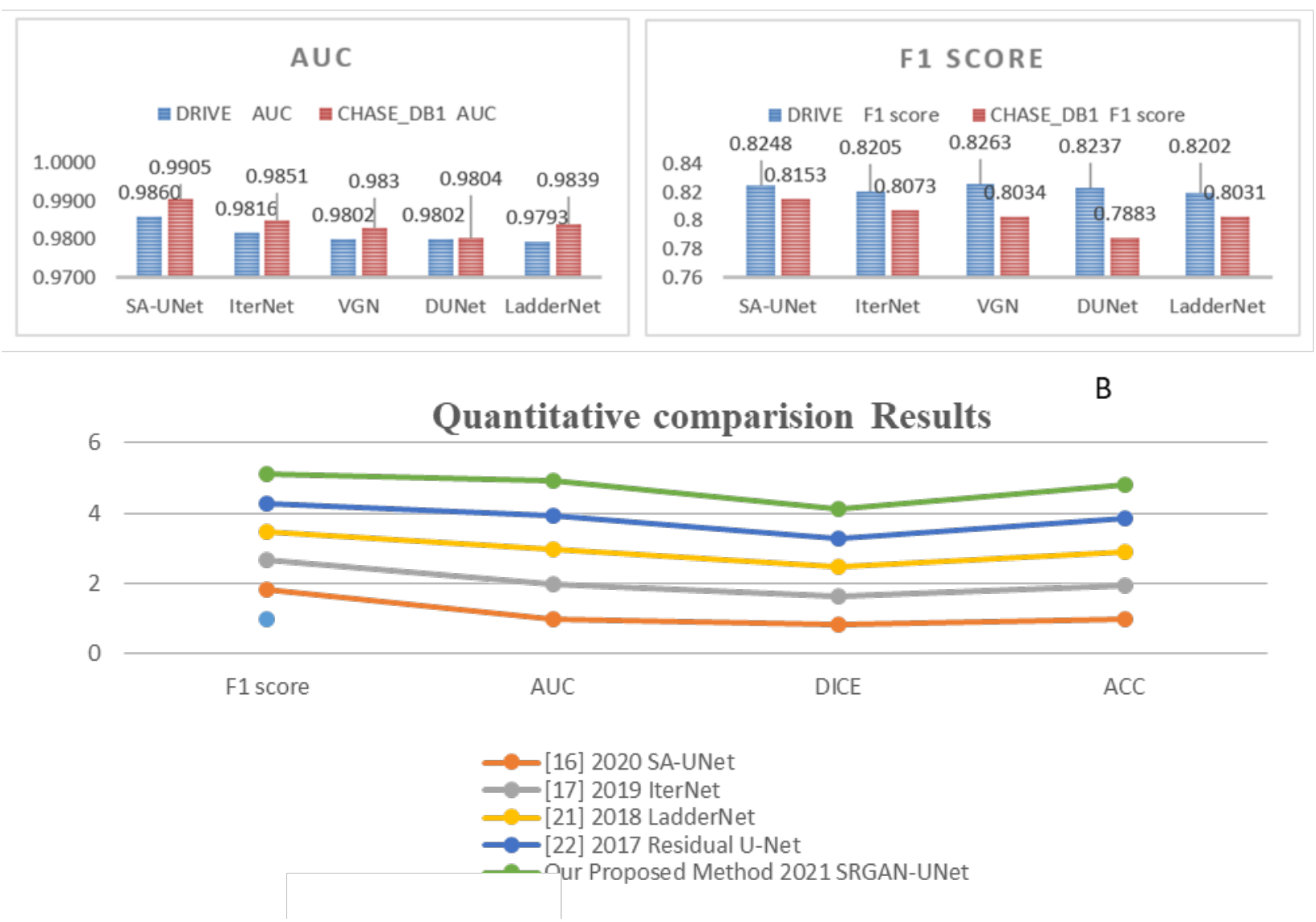

Fig 3. (A) Graphical representation of DRIVE and CHASE_DB1 of different methods, (B). Quantitative result of our proposed method compare from previous methods 
Table 2. Quantitative comparison of performance of our proposed model from previous model on DRIVE dataset

\begin{tabular}{lllllllll}
\hline Method & Year & Model & Sensitivity & Specificity & F1 score & AUC & DICE & ACC \\
\hline$(17)$ & 2020 & SA-UNet & 0.8225 & 0.9824 & 0.8248 & 0.9860 & 0.8263 & 0.9694 \\
$(18)$ & 2019 & IterNet & 0.7791 & 0.9831 & 0.8205 & 0.9816 & 0.8218 & 0.9574 \\
$(15)$ & 2018 & LadderNet & 0.7856 & 0.981 & 0.8031 & 0.9839 & 0.8202 & 0.9561 \\
$(16)$ & 2017 & Residual U-Net & 0.7792 & 0.9813 & 0.8149 & 0.9779 & 0.8171 & 0.9556 \\
Our Proposed Method & 2021 & SRGAN-UNet & 0.8352 & 0.9849 & 0.8457 & 0.9943 & 0.8315 & 0.9736 \\
\hline
\end{tabular}

\subsection{Results}

Figure 4 Left side column we shown Comparison of segmentation masks from fully trained segmentation networks between standard data augmentation and generated DRIVE dataset with SRGAN based UNet network model.

The proposed SRGAN-UNet method was prepared utilizing patches of a size $32 \star 32$ pixels. This fix size gave the best exactness of pixel arrangement by U-Net. Each resolution is acquired by arbitrarily choosing its middle inside the full image. A bunch of 190,000 patches is acquired by arbitrarily separating 9,500 patches in every one of the 20 DRIVE training images. Albeit the patches cover, for example various patches may contain same piece of the original images, no further data increase is performed. The main $90 \%$ of the dataset is utilized for training (171,000 patches), while the last $10 \%$ is utilized for testing (19,000 patches).

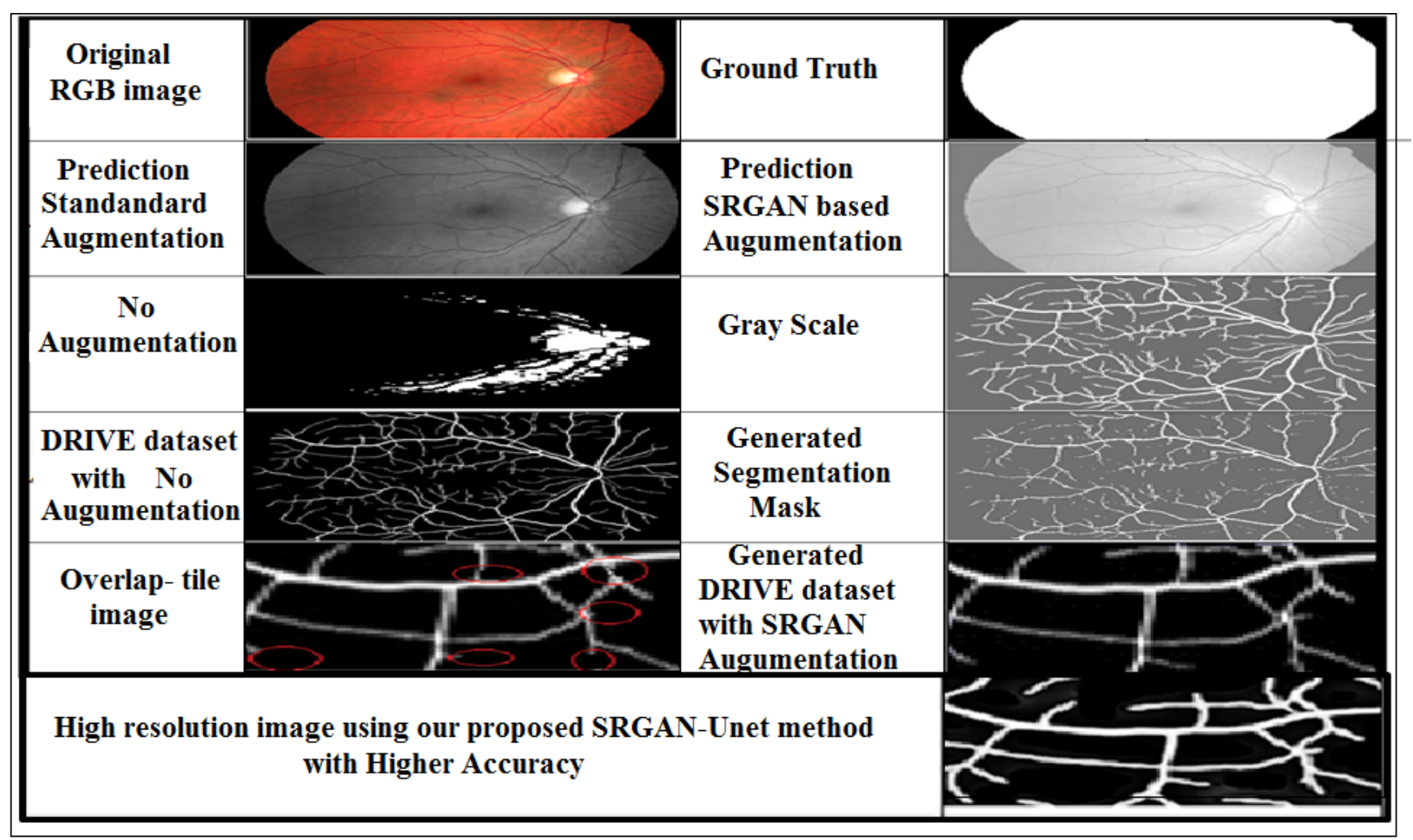

Fig 4. Comparison of retinablood vessel segmentation from fully trained segmentation networks betweenstandard data augmentation and SRGAN-UNetcombine with overlap-tile technique with high resolution image

\section{Conclusion}

In this paper, improved training retina blood vessel datasets with higher resolution images and reduce segmentation loss using SRGAN-U Net method. The proposed method demonstrated to be really critical in data augmentation. We improved the accuracy, affectability and explicitness of SRGAN-UNet method. We additionally applied overlap tile technique for validation 
which made it conceivable to segment high resolution with overall precision $0.9736 \%$.

As Future research work, the technical challenge is to create a mirror camera that can obtain retinal fundus photos automatically lacking requiring the help of an extra lens. Imaging technology in this area is progressing fast and it is likely that in the next few years such cameras may be available. The ability to process multiple computer vision tasks without substantial latency requires more powerful logical boards than what is broadly accessible.

\section{Acknowledgement}

The authors wish to thank the Bishop Heber College, Bharathidasan University, Trichy support. The present study was done in satisfaction of the requirements for- $\mathrm{PhD}$ degree.

\section{References}

1) Park KB, Choi SH, Lee JY. M-GAN: Retinal Blood Vessel Segmentation by Balancing Losses Through Stacked Deep Fully Convolutional Networks. IEEE Access. 2020;8:146308-146322. Available from: https://dx.doi.org/10.1109/access.2020.3015108.

2) Athira S, Francis F, Raphel R, Sachin NS, Porinchu S, Francis S. Smart mirror: A novel framework for interactive display. 2016 International Conference on Circuit, Power and Computing Technologies (ICCPCT). 2016;p. 1-6. doi:10.1109/ICCPCT.2016.7530197.

3) Fu H, Xu Y, Lin S, Wong DWK, Liu J. DeepVessel: Retinal Vessel Segmentation via Deep Learning and Conditional Random Field. Medical Image Computing and Computer-Assisted Intervention - MICCAI 2016. 2016;p. 132-139. Available from: https://doi.org/10.1007/978-3-319-46723-8_16.

4) Akram MU, Atzaz A, Aneeque SF, Khan SA. Blood Vessel Enhancement and Segmentation Using Wavelet Transform. 2009 International Conference on Digital Image Processing. 2009;p. 34-38. doi:10.1109/icdip.2009.70.

5) Gu Z, Cheng J, Fu H, Zhou K, Hao H, Zhao Y, et al. CE-Net: Context Encoder Network for 2D Medical Image Segmentation. IEEE Transactions on Medical Imaging. 2019;38(10):2281-2292. Available from: https://dx.doi.org/10.1109/tmi.2019.2903562.

6) Srivastava A, Valkov L, Russell C, Gutmann MU, Sutton C. VeeSRGAN: reducing mode collapse in SRGANs using implicit variational learning. Neural Data Processing Systems. 2017;p. 3308-3318. Available from: https://arxiv.org/abs/1705.07761.

7) Chen X, Xu C, Yang X, Song L, Tao D. Gated-GAN: Adversarial Gated Networks for Multi-Collection Style Transfer. IEEE Transactions on Image Processing. 2019;28(2):546-560. Available from: https://dx.doi.org/10.1109/tip.2018.2869695.

8) Martin M, Arjovsky, Soumith, Chintala L, Léon, Bottou. Wasserstein S Generative Adversarial Networks. Proceedings of the 34th International Conference on Machine Learning. 2017;p. 214-223. Available from: https://proceedings.mlr.press/v70/arjovsky17a.html.

9) Guibas JT, Virdi TS, Li PS. Synthetic Medical Images from Dual Generative Adversarial Networks. Synthetic medical Images from dual generative adversarial networks. 2017. Available from: https://arxiv.org/abs/1709.01872.

10) Fan Z, Lu J, Wei C, Huang H, Cai X, Chen X. A Hierarchical Image Matting Model for Blood Vessel Segmentation in Fundus Images. IEEE Transactions on Image Processing. 2019;28(5):2367-2377. Available from: https://dx.doi.org/10.1109/tip.2018.2885495.

11) Jin Q, Meng Z, Pham TD, Leyi QC, Wei R, Dunet. DUNet: A deformable network for retinal vessel segmentation. Knowledge Based System. 2019;178:149162. Available from: https://doi.org/10.1016/j.knosys.2019.04.025.

12) Staal J, Abramoff MD, Niemeijer M, Viergever MA, van Ginneken B. Ridge-Based Vessel Segmentation in Color Images of the Retina. IEEE Transactions on Medical Imaging. 2004;23(4):501-509. Available from: https://dx.doi.org/10.1109/tmi.2004.825627.

13) Owen CG, Rudnicka AR, Mullen R, Barman SA, Monekosso D, Whincup PH, et al. Measuring Retinal Vessel Tortuosity in 10-Year-Old Children: Validation of the Computer-Assisted Image Analysis of the Retina (CAIAR) Program. Investigative Opthalmology \& Visual Science. 2009;50(5):20042004. Available from: https://dx.doi.org/10.1167/iovs.08-3018.

14) Hoover $\mathrm{AD}$, Kouznetsova V, Goldbaum M. Locating blood vessels in retinal images by piecewise threshold probing of a matched filter response. IEEE Transactions on Medical Imaging. 2000;19(3):203-210. Available from: https://dx.doi.org/10.1109/42.845178.

15) Azad R, Asadi-Aghbolaghi M, Fathy M, Escalera S. Bi-Directional ConvLSTM U-Net with Densley Connected Convolutions. arXiv. 2019. Available from: https://arxiv.org/abs/1909.00166.

16) Zhang Z, Liu Q, Wang Y. Road Extraction by Deep Residual U-Net. IEEE Geoscience and Remote Sensing Letters. 2018;15(5):749-753. Available from: https://dx.doi.org/10.1109/lgrs.2018.2802944.

17) Guo C, Szemenyei M, Yi Y, Wang W, Chen B, Fan C, et al. Spatial Attention U-Net for Retinal Vessel Segmentation. IEEE. 2021;p. 1236-1242. doi:10.1109/ICPR48806.2021.9413346.

18) Li L, Verma M, Nakashima Y, Nagahara H, Kawasaki R. IterNet: Retinal Image Segmentation Utilizing Structural Redundancy in Vessel Networks. IEEE Applications of Computer Vision. 2020. doi:10.1109/wacv45572.2020.9093621.

19) Shin SSY, Lee D, Yun ID, Lee KM. Deep Vessel Segmentation By Learning Graphical Connectivity. Medical Image Analysis. 2018. Available from: https://doi.org/10.1016/j.media.2019.101556.

20) Zhuang J. LadderNet: Multi-path networks based on U-Net for medical image segmentation. Multi-path networks based on U-Net for medical image segmentation",arXiv. 2018. Available from: https://arxiv.org/abs/1810.07810. 